\title{
Manic Psychosis
}

National Cancer Institute

\section{Source}

National Cancer Institute. Manic Psychosis. NCI Thesaurus. Code C35166.

The manifestation of psychotic symptoms, such as hallucinations or delusions, in the presence of mania. 